Gastroenterologe 2019 $14: 4-5$ https://doi.org/10.1007/s11377-018-0308-z Online publiziert: 29. Oktober 2018 (c) Springer Medizin Verlag $\mathrm{GmbH}$, ein Teil von Springer Nature 2018, korrigierte Publikation 2019

CrossMark

\section{Stefan Zeuzem ${ }^{1}$ - Jürgen F. Riemann ${ }^{2}$}

'Zentrum für Innere Medizin, Medizinische Klinik 1, Universitätsklinikum Frankfurt a. M., Frankfurt a. M. Deutschland

${ }^{2}$ ehem. Medizinische Klinik C, Klinikum Ludwigshafen, Stiftung LebensBlicke, Ludwigshafen, Deutschland

\title{
Neue Wege in der Darmkrebsprävention
}

Die Darmkrebsprävention ist in Deutschland erst spät ein Erfolgsmodell geworden. Obwohl es seit 1977 in der Regelversorgung das Angebot eines Stuhltests auf okkultes Blut gibt, ist über viele Jahre diese Möglichkeit nie richtig angenommen geschweige denn begleitend evaluiert worden. Erst seit 2002, als auch die Koloskopie als eine Option angeboten wurde, konnte ein besserer Zugang verzeichnet werden. Als Qualitätskriterium war auch eine Dokumentation vorgesehen, die endlich Zahlen und Fakten von den koloskopierenden Ärzten ermöglichte. Das Zentralinstitut für die kassenärztliche Versorgung in der Bundesrepublik Deutschland hat diese Daten erfasst und in seinen jährlichen Berichten die Entwicklung öffentlich gemacht. Trotz des Angebots von Stuhltest und/oder Koloskopie sind die aktuellen Zahlen über Inzidenz und Mortalität an Darmkrebs immer noch erschreckend hoch, wenngleich seit 2002 ein signifikanter Rückgang zu registrieren ist.

Der Wissenszuwachs in der Medizin wird immer größer, die Halbwertszeit des medizinischen Wissens immer kürzer; so nimmt es nicht wunder, dass auch in der Darmkrebsprävention neue Wege beschritten werden. Inzwischen hat der nutzerfreundlichere immunologische Stuhltest Einzug in die gesetzliche Krankenversicherung gehalten. Er muss nur einmalig ausgegeben werden, ist bezüglich seiner Sensitivität deutlich besser als der alte guajakbasierte Stuhltest und bedarf keinerlei Diätvorschriften. Die Arbeitsgruppe um Hermann Brenner hat sich durch viele epidemiologische Untersuchungen um die Datenerfassung der Darmkrebsprävention außerordentlich verdient ge- macht. So ist auch ihr Beitrag besonders informativ, da er die Fortschritte mit der Einführung des FIT als nichtinvasivem, niederschwelligem Test beleuchtet und aufzeigt. Die Arbeitsgruppe weist erneut nachdrücklich darauf hin, dass jeder positive Test durch Koloskopie abgeklärt gehört, eine Aussage, die man nicht oft genug wiederholen kann.

Aber auch Stuhltests haben ihre begrenzte Akzeptanz. Von daher werden in Zukunft sicher blutbasierte Tests breiten Einzug in die Prävention halten. Der Nachweis zirkulierender TumorDNA sowie molekulare Signaturen von Krebsen und ihren Vorläufern werden entscheidende Vorteile bringen. Was nach dem FIT kommt, beschreiben Drachsler und Trojan in einem sehr zukunftsorientierten Kapitel.

Ein wichtiger Pfeiler jeder Prävention ist die informierte Entscheidung. Immerhin geht es beim Screening in der Regel um gesunde Menschen, die wissen müssen, welche Chancen sie erwarten und welches Risiko sie mit Vorsorge eingehen. Die Arbeitsgruppe um Klaus Koch vom Institut für Qualität und Wirtschaftlichkeit im Gesundheitswesen (IQWiG) zeigt auf, wie diese informierte Entscheidung entwickelt worden ist. Evidenzbasierte Information ist ein Schlüssel. Das IQWiG wird im Auftrag des gemeinsamen Bundesausschusses (G-BA) bei dem in Zukunft geplanten Einladungsverfahren zur Darmkrebsprävention die bundesweite Information über entsprechende Flyer übernehmen. Von daher ist dieser Beitrag ein wichtiger Einblick in die Frage, wie eine solche Entscheidung aussehen muss.
Mit zunehmender Erkenntnis über die Pathogenese und die Ursache des Kolonkarzinoms wissen wir auch mehr über Risikogruppen. Neben dem erblichen Darmkrebs ist es vor allem die familiäre Häufung, die einer besonderen Aufmerksamkeit bedarf. Für diese risikoadaptierte Früherkennung gibt es inzwischen auch klare Vorstellungen. Dieter Schilling beschreibt, welche Konsequenzen das für Betroffene haben kann. Die Stiftung LebensBlicke stellt ein Konzept zur Diskussion, das medizinisches Fachpersonal so qualifiziert, dass es in der Informationsübermittlung gerade bei Risikopatienten vor allem in Landarztpraxen mitwirken kann. Erfahrungen aus der FAMKOL-Studie haben gezeigt, dass das hervorragend möglich ist.

Im vorletzten Beitrag geht es um die Frage, ob das Geschlecht bei der Aufklärung eine Rolle spielt. Auch hier weisen Untersuchungen aus der Mannheimer Arbeitsgruppe um Sebastian Belle, unterstützt durch die Stiftung LebensBlicke, daraufhin, dass in der Tat die differenzierte Ansprache ein wichtiger Pfeiler sein kann, um Darmkrebsprävention zu propagieren. Genderspezifische Information sollte heute eine Conditio sine qua non sein.

Dieses Schwerpunktheft schließt ab mit einem ganz aktuellen Beitrag von Spitzenvertretern der kassenärztlichen Bundesvereinigung und des GKVSpitzenverbandes zum Krebsfrüherkennungsregistergesetz (KFRG), das bereits 2013 beschlossen worden ist und jetzt, 2019, in die Regelversorgung eingeführt wird. Das KFRG ist ein entscheidender Schritt im Sinne eines Paradigmenwechsels in der Darmkrebsprävention: 
vom opportunistischen Screening hin $\mathrm{zu}$ einem Einladungsverfahren für alle Anspruchsberechtigten. Besonders hervorzuheben ist die Einladung der Männer zur Koloskopie schon ab dem 50. Lebensjahr, da das Erkrankungsrisiko dieser Personengruppe höher ist als das der Frauen, eine Tatsache, auf die die Stiftung LebensBlicke schon seit langem aufmerksam gemacht hat. Es ist zu hoffen, dass mit dem neuen Darmkrebsscreening nicht nur eine Teilnahmesteigerung erzielt, sondern auch eine bessere und ausgewogene Information der Versicherten erfolgen wird.

Wir hoffen, dass wir mit diesem Schwerpunktheft einen Einblick in aktuelle Entwicklungen in der Darmkrebsprävention geben konnten, und wünschen viel Information und Spaß beim Lesen.

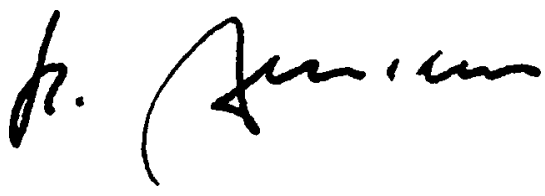

Prof. Dr. Stefan Zeuzem

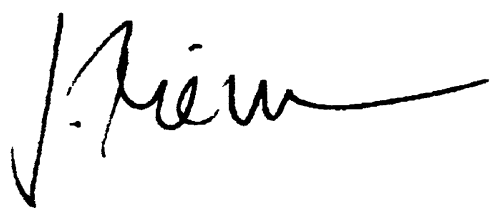

Prof. Dr. Jürgen F. Riemann

\section{Korrespondenzadresse}

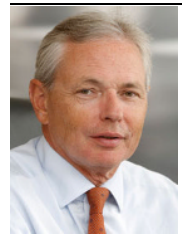

Prof. Dr. Stefan Zeuzem

Zentrum für Innere Medizin,

Medizinische Klinik 1,

Universitätsklinikum

Frankfurt a.M.

Theodor-Stern-Kai 7, 60590 Frankfurt a. M.,

Deutschland

zeuzem@em.uni-frankfurt.de

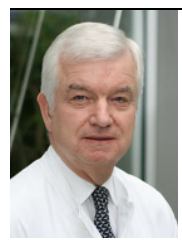

Prof. Dr. Jürgen F. Riemann ehem. Medizinische Klinik C, Klinikum Ludwigshafen, Stiftung LebensBlicke Parkstr. 49, 67061 Ludwigshafen, Deutschland riemannj@garps.de

Interessenkonflikt. S. Zeuzem und J.F. Riemann geben an, dass kein Interessenkonflikt besteht.

\section{(a) \\ LebensBlicke \\ Stiftung Früherkennung Darmkrebs \\ Veranstaltungen der Stiftung LebensBlicke}

Auch im Jahr 2019 lädt die Stiftung LebensBlicke wieder zu interessanten Veranstaltungen rund um das Thema Darmkrebsvorsorge ein:

14.02.2019

Auftakt-Pressekonferenz zum Darmkrebsmonat März 2019 in Ludwigshafen

\subsubsection{9}

Präventionsnachmittag in Kooperation mit dem Rotary Club, der Bürgerstiftung und dem Ernst-Bloch-Zentrum im EBZ in Ludwigshafen

16.03.2019

Benefizkonzert mit der Sängerin Pe Werner in der Friedenskirche Ludwigshafen

Tickets unter www.reservix.de

24.05.2019

Charity-Golfturnier zugunsten der Stiftung LebensBlicke in Leipzig

Die Stiftung LebensBlicke setzt sich seit 1998 für die umfassende Information der Bevölkerung über die Möglichkeiten der Darmkrebsfrüherkennung ebenso wie für die Motivation zur Teilnahme an den angebotenen Maßnahmen ein. Sie leistet dies besonders durch Öffentlichkeitsarbeit in Print- und elektronischen Medien, bundesweite Veranstaltungen über ein Netz von Regionalbeauftragten und durch intensive Teilnahme am zentralen Darmkrebsmonat März eines jeden Jahres.

\section{Stiftung LebensBlicke}

Geschäftsstelle

Schuckertstraße 37

67063 Ludwigshafen

stiftung@lebensblicke.de 